\title{
MANAGING CLASSIFIED DOCUMENTS IN A RELATIONAL DATABASE
}

\author{
Adrian Spalka \\ Department of Computer Science III, University of Bonn \\ Roemerstrasse 164, D-53117 Bonn, Germany \\ adrian@cs.uni-bonn.de
}

\begin{abstract}
The handling of classified paper-based documents follows well established mandatory security policies and the mandatory access control model of Bell and LaPadula convincingly demonstrates how to implement these policies in operating systems. In view of the many difficulties encountered by SeaView's attempt to provide full support for these policies in relational databases, this work pursues the less ambitious aim to use a relational database only for the management of classified documents in concordance with mandatory security policies. In the first part we present the options for the conceptual design of the database, ie the database scheme and the adaptation of the data manipulation language. In the second part we address operational aspects related to the possible use of the database by untrustworthy programs. This investigation yields valuable hints to the solution of the more complex problem of constructing a complete multi-level relational database with sound static and dynamic semantics.
\end{abstract}

Key words: Databases, Security, Confidentiality, Integrity, Logic

\section{INTRODUCTION}

Most commercial companies, government agencies and the military possess classified documents. The term classified states that the content of the document and often also its existence are subject to confidentiality requirements. If a document is marked as classified, then only a specific group of 
persons is allowed to have access to it and it must be kept secret from all other persons.

Since not all classified documents should be kept secret from the same group of persons it is common practice to assign the persons and the documents to named groups and to establish a correspondence between the groups of persons and the groups of documents. If the document group $D$ is in correspondence to the person group $P$, then the members of $P$ are authorised to have access to the documents in $D$ and, which is the main requirement of all mandatory security policies concerned with confidentiality, the documents in $D$ should be kept secret from all persons not in $P$. The document groups express the documents' degree of sensitivity, often also denoted as degree or level of confidentiality; one says that document $A$ has a higher sensitivity than document $B$ if $A$ should be kept secret from more persons than $B$ and, accordingly, fewer persons are authorised to have access to $A$ than to $B$. The person groups, on the other hand, express the persons' degree of trustworthiness, sometimes also denoted as clearance; one says that person $X$ has a higher trustworthiness than person $Y$ if $X$ is authorised to have access to more documents that $Y$ and, accordingly, fewer documents should be kept secret from $X$ than from $Y$.

The handling of classified paper-based documents often follows well established mandatory security policies. In government institutions and the military the following two rules stated in [8] capture the essence of these policies:

The dissemination of information of a particular security level (including sensitivity level and any compartments or caveats) to individuals lacking the appropriate clearances for that level is prohibited by law.

and:

When a document is not in a safe, it is in the custody of some individual trusted not to distribute it improperly.

We prefer to speak of a mandatory confidentiality policy (MCP), if the main rules, as is the case here, refer only to confidentiality. We call the first rule the Primitive Mandatory Requirement (PMR) for it expresses the basic confidentiality requirement, which is the main concern of the policy. The second rule asserts that authorisations to have access to documents are granted in full compliance with the PMR; we call it the Trustworthiness Assumption (TWA).

The PMR has a purely declarative style. It states only that a classified document A should be kept secret from a person $X$, if $X$ 's clearance is insufficient with respect to $A$ 's sensitivity. It does not tell us how to enforce confidentiality of $A$ in front of $X$ and it does not tell us which actions disseminate information about $A$ to $X$. The TWA has a purely declarative style, too. It takes that those authorised to have access to a document will keep it secret 
from others im compliance with the PMR and, by far more important, that they know how to do it.

This work has the following objective: Find a constructive interpretation of the PMR in the context of a relational database that manages only classified documents.

One can be tempted to say that this objective is not very challenging. However, we show that this database retains the sound semantics of relational databases and enforces confidentiality. In view of the troubles encountered by SeaView, it preserves the semantic properties of integrity constraints and never encounters 'polyinstantiation'. We also take a look at extensions to this database that are prone to polyinstantiation. We use the examination to show, however, that sound semantics can be preserved and confidentiality still enforced if we if allow the database not only to withhold data but also to return bogus data. We therefore believe that the findings of this paper provide valuable hints for the construction of a full relational database with the ability to enforce confidentiality - which, of course, by the definition of a relational database implies the preservation of integrity.

The subsequent section comments on some previous works. The main investigation is presented in section 3. Lastly, a conclusion summarises our results.

\section{PREVIOUS AND RELATED WORK}

Security-level-based mandatory controls of information dissemination were originally developed for information recorded on paper. Today the security model developed by Bell and La Padula ([1]) is generally accepted as the standard formalisation of the mandatory security policy. Since the establishment of this model's non-disclosure properties is attributed to a large extent to two of its rules (which are called properties), the Simple-SecurityProperty and the *-Property, these two rules are regarded as the core of Bell and La Padula's model. Originally, this model has been developed for operating systems. It protects a system's confidentiality from a wide range of untrustworthy Trojan Horse programs. But gradually the opinion has consolidated that the two core rules are also indispensable prerequisites for guaranteeing non-disclosure in any kind of multi-level database systems - an opinion that needs to be revised in view of the semantic problems that these databases still struggle with.

The success of Bell and La Padula's security model in operating systems encouraged attempts to construct a relational database with the ability to manage classified items with a more complex structure than those of documents. However, the enticing idea that such a database can be defined as the 
plain 'sum' of a relational database and the two rules from Bell and La Padula's security model soon turned out to lead to intricate semantic problems. The multi-level relational data model introduced in [3] and formalised in [4] is claimed to be an extended relational data model suitable for the enforcement of mandatory security. A multi-level relation scheme is defined as $R\left(A_{1}, C_{1}, \ldots, A_{n}, C_{n}\right)$. Each $C_{i}$ defines a range of security levels for the attribute $A_{i}$. The reason for classifying single attributes, according to the authors' opinion, is that an attribute value represents a fact (which is in clear contradiction to the definition of a relational database). In addition to it, a security level is assigned to the whole relation scheme and a tuple's security level is computed from those of its attribute values'. The authors note that: 'Functional dependencies correspond to real-world constraints; that is, they are not just a property of a particular relation instance' and 'The requirements for consistency [at each access class] affect the integrity rules of the relational model, which are formulated as global constraints on an entire database.' Yet their approach to the handling of integrity in this database does not account for these facts. It relies on purely syntactical - obviously inadequate - adjustments instead. The name of the resulting self-made semantic havoc is 'polyinstantiation'.

An approach to a logic foundation of multi-level databases is presented in [16]. The author offers two views: the system-high world and the per-level world views. The author states that 'The system-high world method does not accommodate polyinstantiation ... since each piece of data is assigned only one level...'. In the per-level world, polyinstantiation is allowed, yet its operational treatment does not seem to rest on any fixed semantics. In conclusion, while correctly stressing the necessity of a formal basis for multi-level databases, no concrete advances in this direction are presented.

A similar idea is presented in [7], which regards a multi-level database as a collection of databases. The data of each database is represented as one logical theory of first-order logic. Promising though the authors' observations are, the approach has not been further developed.

\section{CLASSIFIED DOCUMENTS IN RELATIONAL DATABASES}

\subsection{Preliminary considerations}

The essence of mandatory confidentiality policies is the PMR, which specifies confidentiality demands in an informal manner. Its application to an environment involves two steps: a declarative definition of confidentiality 
and an operational translation for its enforcement. This is carefully done for operating systems managing classified documents by [1] and for general systems defined through a sequence of inputs and outputs by, eg, [6].

In a computer system, the common understanding of the confidentiality demand stated in the PMR is that a secret item is kept confidential if the user gets no information from the system on it. Translated to prohibitions, we must ensure that:

1. the user does not get the secret data itself or any part of it

2. the data the user gets from the system must not depend or be influenced by secret data

Point (1) prevents that a user gets any secret data directly. Point (2) expresses two concerns; it prevents that:

- the user learns of the secret data's existence

- the user gets secret data from a Trojan horse, ie an untrustworthy program

We can translate these prohibitions into the following positive requirements:

The result of an operation executed by the user

(P1) may comprise only data he is authorised to get and

(P2) may refer only to items he is authorised to get.

Our aim in this paper is the enforcement of confidentiality of classified documents stored in and managed by a relational database. We approach this task at the declarative level and the operational level. The declarative level defines data structures and operations on them; it has no notion of programs and, therefore, is not concerned with untrustworthy ones. The operational level deals with implementation details and the particularities of a computer; it is this level that can take untrustworthy programs into account.

A classified document consists of two parts, a name and content, with the following structure:

- document name: (security level, name)

- document content: (reference to a document name, content)

In a database the user can retrieve data with the select operation and he can modify data with the insert, delete and update operations. The select operation refers only to relations and its result is a, possibly empty, answer set. The modifying operations refer to relations and to integrity constraints. Their result is a yes/no-answer, which is dictated by the integrity constraints.

Therefore, the satisfaction of the PMR in our database has the following interpretation:

(P3) Confidentiality of the document parts is enforced, if the evaluation of a select command submitted by the user refers to and returns only data, ie document parts or results of functions applied to them, he is authorised to access (covers both the declarative and operational part of (P1)). 
(P4) Confidentiality of the document's existence is enforced, if the computation of the answer to a modifying command submitted by the user refers only to document parts he is authorised to access (covers the declarative part of (P2)).

(P5) Given that the user has chosen a fixed security level for the current session, a Trojan horse cannot reveal secret data if a modifying command submitted by the user modifies only document names with the session's security level and document contents the referred names of which have the session's security level (covers the operational part of (P2)).

Thus, the procedure applied to a command submitted by the user comprises the following steps:

1. Tighten the conditions for selecting tuples so that only tuples he is authorised to access are selected for processing (applies to select, delete and update operations)

2. Ensure that integrity constraints need to evaluate only tuples he is authorised to access in order to yield the answer defined by their semantics (applies to modifying operations)

Note that point (2) implies that we do not tolerate a violation of integrity.

To construct our database we employ a preventive design. It prevents a loss or damage under the assumption that a user's clearance (and, consequently, the rights granted to him) reflects his actual trustworthiness and that the degree of sensitivity assigned to a document is based on its actual confidentiality. We do not address restorative support, which provides measures, by nature of the problem, to detect and, if possible, to restore a loss or damage resulting from misuse of granted rights by a corrupt user (an insider influenced by bribery or the like).

We assume that a set of security levels, $S L$, is given on which a partial order ' $\leq$ ' is defined. The SQL examples in the subsequent sections were tested on the MS SQL Server v7.

\subsection{Approach 1: Grouped attributes}

\subsubsection{Database scheme}

The database scheme needed to represent classified documents is fairly simple.

We have a relation to store document names, CDNames(SL, Name), such that $\mathrm{SL}$ is the security level component of the document name, Name is the name component of the document name, and if a tuple is in the extension of CDNames, then a classified document with the name (SL, Name) exists; otherwise such a document does not exist. Its semantic restrictions are: the 
domain of SL is the set of security levels SL, the domain of Name comprises suitable constants in the database language, eg character strings, and there is a primary key constraint on the pair (SL, Name), ie the combination of both attributes must be unique, which prevents the existence of two documents with identical names.

And we have a relation to store documents' content, CDContent(SL, Name, Content), such that the pair (SL, Name) is a reference to a document with this name, content is the content of the document with the referenced name, and if a tuple is in the extension of CDContent, then the classified document with the referenced name has the content of Content, otherwise the document has no such content. Its semantic restrictions are: the domains of SL and Name are inherited from CDNames, the domain of Content is any suitable data type, there is a foreign key constraint from CDContent(SL, Name) to CDNames(SL, Name), which prevents the existence of anonymous content, there is an (implicit) primary key constraint on all attributes, which prevents the existence of identical contents within a document and an (optional) primary key constraint on the pair (SL, Name), which can be used to ensure that a document has only one content; if defined, it overrides the preceding constraint.

Since the database must make some decisions based on the partial order of the security levels and the users' clearance, we can - as an option - store this information in the database as well. We therefore have three more relations. If a tuple is in the extension of the relation Levels(SL), then SL is an element of the set of security levels SL; otherwise SL has no such element. The partial order ' $\leq$ ' on SL can be represented by its transitive closure in the relation POL(GEL, LEL), such that GEL and LEL are references to security levels and if a tuple is in the extension of POL, then the security level referred to by GEL is greater than or equal to the security level referred to by LEL; otherwise these security levels are unrelated in ' $\leq$ '. Lastly, we store the users and their clearances in the relation Users(Name, Clearance) with obvious semantics. We assume that the database obtains the identity of the user who submits a command to it from a source that previously authenticated the user and that all attributes are defined as NOT NULL.

All restrictions so far defined are semantic, ie they reflect only structural properties of classified documents and are not concerned with confidentiality. Modifications and restrictions needed to enforce the PMR, ie (P3), (P4) and (P5), in the database are examined in subsequent sections.

\subsubsection{Enforcement of confidentiality during selection}

As we will see, access restrictions are all we need to enforce the PMR during selection in a database with this scheme. Access restrictions can be 
expressed in two equivalent ways: by augmenting the user's select-statement with additional conditions (restrictions) or by defining views for the user. In this work we go the first way. We therefore consider a select-statement as it is submitted by a user and its modification that is actually executed.

We approach the general solution by first taking a look at some selectstatements. Our user's name is $U$ and we assume that the database knows the user's name.

In general, select-access to the relations Levels, POL and Users or a subset thereof can be granted or refused in concordance with an organisation's regulations. With respect to the PMR, the user may have select-access to the relations Levels and POL restricted to the subset indicated by his clearance, and in the relation Users select-access only to the tuple identifying himself.

(A) The user wants to retrieve the security levels:

Submitted statement: select SL from Levels

Executed statement in informal notation:

select SL from Levels, Users

where Users.Name = ' $U$ ' and Levels.SL $<=$ Users.Clearance

Executed statement in SQL:

select SL from Levels, Users where Users. Name = 'U' and

Levels.SL in (select LEL from POL where GEL = Users.Clearance)

(P3) and (P4) are not affected since the selection does not refer to any part of a classified document. However, the condition in the where clause shows that we can easily restrict the user's access to those security levels he is authorised to use.

We do not need to consider (P5) during selection since a Trojan Horse can only pass data in a modifying operation.

(B) The user wants to retrieve the names and content of classified documents

Submitted statement:

select * from CDNames, CDContent

where CDNames.ID = CDContent.Ref_ID

Executed statement in informal notation:

select CDNames.SL, CDNames.Name, CDContent.Content

from CDNames, CDContent, Users

where CDNames.ID $=$ CDContent.Ref_ID and

Users. Name = 'U' and CDNames.SL $<=$ Users. Clearance

We immediately see that both (P3) and (P4) are met. The condition in the where clause confines the reference to document names the user is authorised to access and, thus, the answer set comprises this set only or a subset of it.

(C) Conclusions 
The satisfaction of the PMR for classified documents in a database is easily accomplished during selection.

Suppose that we want to admit document content parts with an individual security level. Then the select statements examined in C) and, in particular, in D) indicate that the security level of such a part should not be less than or incomparable to the security level attached to the document's existence. Due to (P3) and (P4) access to these parts is governed by the security level attached to the document's existence, which implicitly raises the security level of such a part to that of the document's existence.

\subsubsection{Enforcement of confidentiality during insertion}

All modifying operations modify a single relation only. However, they refer to the specified relation and to integrity constraints, which are affected by the intended modification. Semantic restrictions defined as integrity constraints are automatically evaluated by the database. We express further restrictions implied by the PMR as additional conditions in the where-clause or as triggers. We assume that the user has no modifying-access to the relations Levels, POL and Users.

The insert-statement does not comprise conditions for the insertion. Thus, it cannot be augmented and there is no distinction between the submitted statement and the executed one. If the insert-statement comprises a selectpart, then the presented conditions on selection apply, too.

(A) The user wants to insert a document name

Submitted/executed statement:

insert into CDNames (SL, Name) VALUES (v1, v2)

Triggers: T1 Checks that the user specified a security level authorised for his clearance.

Informal notation:

if (not (Clearance of $U>=v 1)$ ) then

print 'Invalid security level. Insertion failed.'

rollback transaction

end if

SQL:

create trigger $\mathrm{T} 1$ on CDNames for insert as

declare@L varchar(20)

select @L=SL from inserted

if @L not in (select POL.LEL from POL, Users

where Users.Name = ' $U$ ' and GEL $=$ Users. Clearance)

begin

print ('Invalid security level. Insertion failed.') 


\section{rollback transaction \\ end}

Affected integrity constraints:

Primary key on CDNames. This constraint performs the following check: if $(v 1, v 2)$ in (select SL, Name from CDNames) then

print 'Primary key violation. Insertion failed.'

rollback transaction end if

It can possibly violate (P4) since the select statement refers to all document names. But suppose that the trigger $\mathrm{T} 1$ executes before this constraint is checked. If T1 rolls the insertion back, then there is no need to check this constraint. If, on the other hand, $\mathrm{T} 1$ is successfully passed, then its check ensures that $\mathrm{v} 1$, the security level specified by the user, is authorised. And, hence, the check of the primary key constraint can be restricted to tuples with $\mathrm{SL}=\mathrm{vl}$. Without affecting the constraint's semantics, we can now rewrite its procedure as follows:

if $\mathrm{v} 2$ in (select Name from CDNames where $\mathrm{SL}=\mathrm{v} 1$ ) then print 'Primary key violation. Insertion failed.'

rollback transaction end if

If this check is passed, then the user gets the information that the specified document name does not exist. If it fails, then the user is notified of a name conflict the reason of which is a document he is authorised to access. In conclusion, this constraint meets (P4) if it $\mathrm{T} 1$ is executed first.

Foreign key to Levels: If passed, the trigger T1 ensures that this constraint never fails, ie, in effect, it is redundant for insert-operations.

Foreign key from CDContent: By definition, foreign key constraints never fail on insertions to the referenced relation.

Note that the satisfaction of (P4) depends on the order of execution: triggers must be evaluated before integrity constraints are checked. The MS SQL Server (used here to check the examples) first checks integrity constraints and then evaluates triggers. In this case another solution must be employed, eg, we can drop the integrity constraints from the table definition and insert their checks in our triggers.

To meet the operational requirements stated in (P5), we must assume that the insertion command is possibly submitted by a Trojan horse. To satisfy (P5) the database can either accept only the Name attribute in the insert command and supply the user's session security level for the SL attribute, or we modify the trigger $\mathrm{T} 1$ as follows:

if (not (session security level of $U=v 1)$ ) then print 'Invalid security level. Insertion failed.' 
rollback transaction

end if

(B) The user wants to insert a document content

This operation turns clumsy is we use surrogate-attributes and do not want the user to know their values. To insert the content of a document the user must also enter a reference to an existing document name. If we do not use surrogate-attributes, then the reference is the document name itself (which comprises both the security level and the name); if we use surrogateattributes, then the reference is the surrogate value corresponding to the document name. Thus, if we want to allow the user to use the SQL insertstatement for performing this operation, then we must choose one of these options. From the viewpoint of semantics both options are equivalent: to insert the content of a document the user must know its name.

Submitted/executed statement:

insert into CDNames (Ref_ID, Content) VALUES (v1, v2)

Triggers. T2 Checks that the user specified a Ref_ID value corresponding to a document name he is authorised to select. Informal notation:

select SL from CDNames where ID = v1

if (not (Clearance of $U>=S L)$ ) then

print 'Invalid reference ID. Insertion failed.'

rollback transaction

end if

Affected integrity constraints.

Foreign key to CDNames: It can possibly violate (P4) since it can check references to all document names. But, again, suppose that the trigger T2 executes before this constraint is checked. The check that the Ref_ID specified by the user corresponds to a document name he is authorised to access implies a check that the document name exists, which the foreign key constraint performs, too. Thus, if successfully passed, the trigger T2 ensures that this constraint never fails, ie, in effect, it is redundant for insert-operations, too.

Primary key on Ref_ID: This constraint performs the following check:

if $\mathrm{v} 1$ in (select Ref_ID from CDContent) then

print 'Primary key violation. Insertion failed.'

rollback transaction

end if

It can possibly violate (P4) since the select statement refers to all document references. However, if T2 rolls the insertion back, then it is not executed. Suppose that T2 is passed. If the check of this constraint is also passed, then the user is notified that content for the referenced document does not exist, and if it fails, then the user is notified of a conflict the reason 
of which is a document he is authorised to access. In conclusion, this constraint meets (P4) if it T2 is executed first.

To satisfy (P5) we modify the trigger T2 as follows:

select SL from CDNames where ID $=\mathrm{v} 1$

if (not (session security level of $U=S L)$ ) then

print 'Document name not found. Insertion failed.'

rollback transaction

end if

(C) Conclusions

The satisfaction of the PMR for classified documents in a database is also easily accomplished during insertion.

Suppose, again, that we want to admit document content parts with an individual security level. The insert statement examined in B) indicates that the security level of such a part should not be less than the security level attached to the document's existence. Due to (P3) and (P4) access to these parts is governed by the security level attached to the document's existence, ie the document name in our database, which implicitly raises the security level of such a part to that of the document's existence.

But can we assign to a document part a security level that is higher than that assigned to its existence? Suppose that such a part exists. Then the primary key constraint on Ref_ID fails if the user attempts to insert a part with a lower security level. This failure violates (P4) since the reason for it is data that should be kept secret from the user. But we can still admit parts with a higher security level if we find a way for the constraint to fail in concordance with (P4), ie without referring to this part. This can be accomplished with aliases the use of which is formally described, eg, in [15]. Basically, the insertion of a part with a higher security level must be accompanied by an insertion of a bogus part with a lower security level, which the user with a lower clearance cannot modify. His insertion will then fail on the account of the bogus part - it obviates a reference to secret data, ie complies with (P4), and preserves integrity. So the answer to our initial question is yes if allow the database not only to withhold data but also to return bogus data.

We omit the delete and update command from our paper for reasons of space.

\subsection{Approach 2: Structured name-spaces}

The approach presented in the preceding section uses two attributes to represent the composite name of a classified document. If we extend a relational database such that the value of an attribute can be a composite value, ie a tuple in the mathematical sense, then section 3.2 can be rewritten in a semantically cleaner fashion, but the results and findings are still the same. 
The formal grounds for this approach, ie for a database with structured name-spaces, are given in [14] and can be used to elaborate the details in a straightforward manner. We omit it here for reasons of space and redundancy.

\section{CONCLUSION}

This little database is all we need to manage classified documents. Integrity constraints retain their standard meaning and are never violated. And the confidentiality demand stated in the PMR is enforced. We have followed some simple principles in this work:

- Do not use the special NULL value. It has no common meaning in open databases and, therefore, can make reasoning about confidentiality only more messy. Admittedly, this value is often used in practice, however, more for reasons of convenience than necessity. Most schemes can be designed in a way that avoids this value.

- Set the primary key on all identifying attributes and make sure that references to a relation (in both integrity constraints and select commands) use all of them.

- If the primary key constraint is defined on all attributes of a relation, then structured name-spaces can be used to enforce confidentiality in line with integrity.

- If the primary key constraint is defined on a proper subset of a relation's set of attributes, then aliases can be used to enforce confidentiality in line with integrity.

- The same methods can be used to enforce confidentiality in line with foreign key constraints.

Our database preserves the standard database semantics and enforces confidentiality with access restrictions only and a modelling of composite names. But we have also realised that a database with a more complex structure can also enforce confidentiality while preserving semantics if (carefully managed) bogus data are admitted. In view of some prominent problems of SeaView we never need to worry that a trusted user is confused ([17]), we do not have to guess about versions, degrees of interest or recency ([4]), nor do we have to deal with imprecise beliefs ([12]).

We intend to pursue this approach and soon present further results on sound databases with the ability to enforce confidentiality. 


\section{REFERENCES}

[1] Bell, David Elliott, and Leonard J. La Padula. (1975) Secure computer system: Unified exposition and multics interpretation. MITRE Technical Report 2997. MITRE Corp, Bedford, MA.

[2] Bonyun, David A. (1980) 'The Secure Relational Database Management System Kernel: Three Years After'. 1980 IEEE Symposium on Security and Privacy. IEEE Computer Society Press. pp 34-37.

[3] Denning, Dorothy E. et al. (1986) 'Views for Multilevel Database Security'. 1986 IEEE Symposium on Security and Privacy. IEEE Computer Society Press. pp 156-172.

[4] Denning, Dorothy E., et al. (1987) 'A Multilevel Relational Data Model'. 1987 IEEE Symposium on Security and Privacy. IEEE Computer Society Press. pp 220-234.

[5] Denning, Dorothy E., et al. (1988) 'The SeaView Security Model'. 1988 Symposium on Security and Privacy. IEEE Computer Society Press. pp 218-233.

[6] Focardi, Ricardo. (1996) 'Comparing Two Information Flow Security Properties.' 9th IEEE Computer Security Foundations Workshop 1996. IEEE Computer Society Press. pp 116-122.

[7] Garvey, Thomas D., Teresa F. Lunt, Xiaolei Qian and Mark E. Stickel. (1992) 'Toward a tool to detect and eliminate inference problems in the design of multilevel databases'. Ed Bhavani M. Thuraisingham and Carl E. Landwehr. Database Security VI. IFIP WG11.3 Workshop on Database Security 1992. Amsterdam: North-Holland, 1993. pp 149-167.

[8] Landwehr, Carl E. (1981) 'Formal Models for Computer Security'. ACM Computing Surveys 13.3:247-278.

[9] Lunt, Teresa F. (1988) 'SeaView'. Ed Teresa F. Lunt. Research Directions in Database Security. 1st RADC Database Security Invitational Workshop 1988. New York et al: Springer-Verlag, 1992. pp 13-32.

[10] Qian, Xiaolei, and Teresa F. Lunt. (1992) 'Tuple-level vs. element-level classification'. Ed Bhavani M. Thuraisingham and Carl E. Landwehr. Database Security VI. IFIP WG11.3 Workshop on Database Security 1993. Amsterdam: North-Holland, 1993. pp 301-315.

[11] Reiter, Raymond. (1984) 'Towards a Logical Reconstruction of Relational Database Theory'. Ed Michael L. Brodie, John Mylopoulos and Joachim W. Schmidt. On Conceptual Modeling. New York: Springer-Verlag. pp 191-238.

[12] Smith, Kenneth, and Marianne Winslett. (1992) 'Entity Modeling in the MLS Relational Data Model'. 18th VLDB Conference. pp 199-210.

[13] Spalka, Adrian. (1996) 'The Non-Primitiveness of the Simple-Security Property and its Non-Applicability to Relational and Logic-Based Databases'. 9th IEEE Computer Security Foundations Workshop 1996. IEEE Computer Society Press. pp 146-155.

[14] Spalka, Adrian, and Armin B. Cremers. (1997) 'Structured name-spaces in secure databases'. Ed T. Y. Lin and Shelly Qian. Database Security XI. IFIP TC11 WG11.3 Conference on Database Security. London et al: Chapman \& Hall, 1998. pp 291-306.

[15] Spalka, Adrian, and Armin B. Cremers. (1999) 'The effect of confidentiality on the structure of databases'. Ed Vijay Atluri and John Hale. Research Advances in Database and Information Systems Security. IFIP TC11 WG11.3 13th Conference on Database Security. Boston et al: Kluwer Academic Publishers. pp 253-268.

[16] Thuraisingham, Bhavani M. (1988) 'Foundations of Multilevel Databases'. Ed Teresa F. Lunt. Research Directions in Database Security. 1st RADC Database Security Invitational Workshop 1988. New York et al: Springer-Verlag, 1992. pp 199-226.

[17] Wiseman, Simon. (1989) 'On the Problem of Security in Databases'. Ed David L. Spooner and Carl. E. Landwehr. Database Security III. IFIP WG 11.3 Workshop on Database Security 1989. Amsterdam: North-Holland, 1990. pp 301-310. 